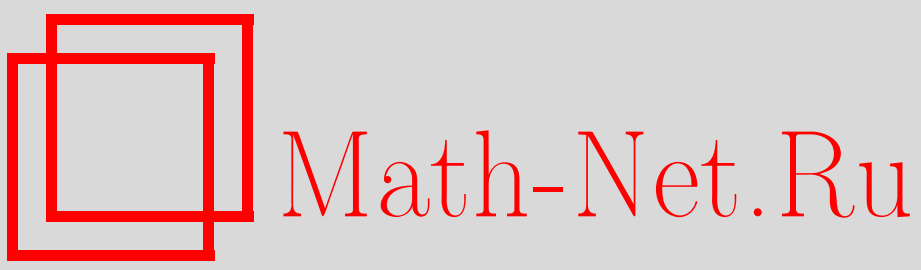

К. И. Сонин, Полупримитивные и полусовершенные кольца лорановских рядов, Матем. заметки, 1996, том 60, выпуск 2, 300-303

DOI: https://doi.org/10.4213/mzm1829

Использование Общероссийского математического портала MathNet.Ru подразумевает, что вы прочитали и согласны с пользовательским соглашением

http://www. mathnet.ru/rus/agreement

Параметры загрузки:

IP : 54.196 .121 .252

26 апреля 2023 г., 09:58:43

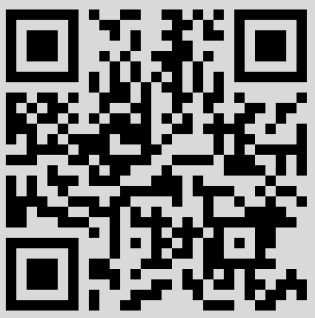




\section{ПОЛУПРИМИТИВНЫЕ И ПОЛУСОВЕРШЕННЫЕ КОЛЬЦА ЛОРАНОВСКИХ РЯДОВ}

\section{К. И. Сонин}

Введение и формулировки основных результатов. Кольца косых лорановских рядов (или, в более общем случае, кольца рядов Мальчева-Неймана) впервые появились в работах, связанных с вложением групповых алгебр в тела (см., например, [1]) и свойствами тел. В этой же связи в [2] рассматривался случай простого артинова кольца коэффициентов. В [3] кольцевые свойства нётеровых колец рядов Лорана использовались для исследования глобальной размерности и размерности Крулля нётеровых РІ-колец. В [4] (для колец рядов Мальцева-Неймана) изучались свойства первичных идеалов, был доказан критерий простоты (доказательство критерия бирегулярности находится в печати), в [5] - регулярность в смысле фон Неймана. В данной работе рассматриваются полупримитивность и полусовершенность колец рядов Лорана, приводятся некоторые достаточные условия примитивности. Основными результатами являются следуюшие теоремы.

ТЕОРема 1. Если $R$ рядов Лорана $R((x, \varphi))$ полупримитивно.

ТЕОРема 3. Если кольчо $R$ полусовершенно, то и кольчо $R((x, \varphi))$ полусовериенно.

Все кольца предполагаются ассоциативньми и обладающими ненулевой единицей.

Определения, обозначения и элементарные свойства. Кольцо ко$c b x$ рядов Лорана $R((x, \varphi))$ над кольцом $R$ с автоморфизмом $\varphi$ определяется (например, в [6, с. 19]) как кольцо, образованное формальными степенными рядами вида

$$
\sum_{i=m}^{\infty} a_{i} x^{i}, \quad m \in \mathbb{Z}, \quad a_{i} \in R
$$

$x$ - независимая переменная, с обычным сложением и умножением, заданньм формулой

$$
\left(\sum_{i=t}^{\infty} a_{i} x^{i}\right)\left(\sum_{j=r}^{\infty} b_{j} x^{j}\right)=\sum_{k=t+r}^{\infty}\left(\sum_{i+j=k} a_{i} \varphi^{i}\left(b_{j}\right)\right) x^{k} .
$$

На протяжении всей работы $T$ будет обозначать кольцо $R((x, \varphi))$. Если $I$ - подмножество кольца $R$, то через $\bar{I}$ обозначается подмножество кольца $T$, состоящее из всех рядов, коэфффициенты которых лежат в $I$. Если $J$ - подмножество кольца $T$, то через $\lambda(J)$ обозначается множество всех элементов кольца $R$, являющихся коэффициентами при младших степенях элементов $J$. Радикал Джекобсона кольца $R$ обозначается $J(R)$. Через $\mathbb{Z}$ обозначается множество целых чисел.

Кольцо $R$ называется правым кольцом Голди, если оно не содержит бесконечных прямых сумм ненулевых правых идеалов и удовлетворяет условию максимальности для правых аннуляторов; полусовершенным, если $R / J(R)$ - полупростое артиново и идемпотенты поднимаются по модулю $J(R) ;$ полупримарным, если $R / J(R)$ - полупростое артиново и $J(R)$ нильпотентен.

(C) К.и. Сонин 1996 
ОПРЕДЕЛЕНИЕ. Пусть $\varphi$ - автоморфизм кольца $R$. Правый (левый, двусторонний) идеал $I$ называется правым (левым, двусторонним) $\varphi$-идеалом, если $\varphi(I) \subseteq I$.

ОПРЕДЕЛЕНИЕ. Колњцо $R$ называется $\varphi$-первичным, если для любых $\varphi$-идеалов $I, J$ кольца $R$ из $I J=0$ следует, что $I=0$ или $J=0 ; \varphi$-примитивным, если в нем найдется максимальный правый идеал, не содержащий ненулевых $\varphi$-идеалов.

Для доказательства теорем нам понадобятся некоторые элементарные свойства лорановских рядов. Утверждения следующей леммы либо хорошо известны, либо проверяются непосредственно.

Лемма 1. (1) Если $f=\sum_{i=t}^{\infty} f_{i} x^{i}$ u $f_{t}$ обратим справа (слева), то $f$ обратим справа (слева) в кольче $R((x, \varphi))$.

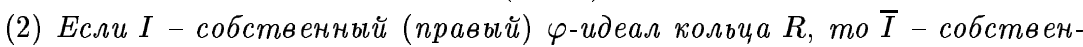
ный (правый) идеал кольчат.

(3) $E$ сли $I-\varphi$-идеал кольча $R, K=R / I$, mо $T / \bar{I} \cong K((x, \varphi))$.

(4) $E с л и I-($ правый) идеал $T$, то $\lambda(I)-($ правый) $\varphi$-идеал $R$.

(5) Если элемент а является коэффициентом некоторого ряда из идеала I кольиа T, то $\varphi^{i}(a)$ является коэффициентом какого-то ряда из I для любого $i \in \mathbb{Z}$.

(6) Если $f=\sum_{i=t}^{\infty} f_{i} x^{i} \in J(T)$, то $f_{t}-$ делитель нуля в $R$.

СледСТВИЕ. Если любой элемент кольиа $R$ коэффициентов есть сумма идемпотента и обратимого элемента, то и любой элемент кольца рядов Лорана $R((x, \varphi))$ представляется в виде суммы идемпотента и обратимого әлемента.

ДокАзАтЕльство. Для произвольного ряда $f=\sum_{i=t}^{\infty} f_{i} x^{i}$ следует, что $f_{t}=e+u$, где $e-$ идемпотент, а $u$ - обратимый элемент в кольце $R$. Теперь ряд $u x^{-t}+\sum_{i=t+1}^{\infty} f_{i} x^{i}$ обратим в $T$ по лемме $1(1)$, и, следовательно, $f=e+u x^{-t}+\sum_{i=t+1}^{\infty} f_{i} x^{i}$ - сумма идемпотента и обратимого элемента.

ЛЕмма 2. $J(T)$ лежит в $\overline{J(R)}$.

ДоКАЗАТЕЛЬСТво. Покажем, что если $I$ - максимальный правый идеал $R$, то $\bar{I}$ - максимальный правый идеал в $T$. Для этого достаточно показать, что $\forall r \in T$ $\exists h \in \bar{I}, g \in T$ такие, что $h+r g=1$. Заметим, что без ограничения общности можно рассматривать только те $r=\sum_{i=0}^{\infty} r_{i} x^{i}$, для которых $r_{0} \neq 0$. Из максимальности $I$ следует, что $\exists a \in I, b \in R$ такие, что $a+r_{0} b=1$, а тогда

$$
a+r b=1+\sum_{i=1}^{\infty} r_{i} \varphi^{i}(b) x^{i}
$$

Теперь существование $f$, такого что $(a+r b) f=1$, следует из леммы $1(1)$, и, значит, $h+r g=1$, где $h=a f \in \bar{I}, g=b f \in T$.

СледствИЕ. Если $J(R)$ нильпотентен, то и $J(T)$ нильпотентен; $\boldsymbol{\theta}$ этом случае $J(T)=\overline{J(R)}$, в частности, если $J(R)=0$, то и $J(T)=0$. 
ДоКАЗАТЕЛЬСТВО. Пусть $(J(R))^{n}=0$, тогда для любого ряда $f$ из $\overline{J(R)}$, любого ряда $g \in T$ выполняется $(f g)^{n}=0$ и, следовательно, элемент $1+f g$ обратим. Таким образом, $\overline{J(R)} \subseteq J(T)$, а обратное включение получается из леммы 2 .

Заметим, что обратное, вообще говоря, неверно: из $J(T)=0$ не следует $J(R)=0$, например, если $R$ является кольцом формальных степенных рядов надполем $(\varphi \equiv 1)$. В этом случае $J(T)=0$ по лемме $1(6)$.

Полупримитивные и примитивные кольца ря дов Лорана. Теорема 1 для колец лорановских рядов является аналогом теоремы 2 в [7] для колец многочленов Лорана.

ДОКАЗАТЕЛЬСТВо ТЕОРЕМЫ 1. Сначала покажем, что в наших условиях кольцо $R$ полупервично. Действительно, пусть $I$ - нильпотентный идеал в $R$, тогда $\varphi^{n}(I)$ нильпотентен для любого $n \in \mathbb{Z}$. Нильпотентные идеалы кольца лежат в первичном радикале, следовательно, идеал $N=\sum_{n \in \mathbb{Z}} \varphi^{n}(I)$ лежит в первичном радикале, который в правом кольце Голди всегда нильпотентен. Таким образом, поскольку идеал $N$ нильпотентен и $\varphi(N)=N$, из $\varphi$-первичности кольца $R$ следует $N=0$, и, следовательно, $I=0$, т.е. $R$ полупервично. Предположим теперь, что утверждение теоремы не выполняется, т.е. $J=J(T) \neq 0$. Тогда $\lambda(J) \neq 0$, и мы покажем, что $\lambda(J)$ существенен как правый идеал в $R$. Если нет, то найдется $M$ - правый идеал кольца $R$ такой, что $\lambda(J) \cap M=0$, и, следовательно, $M \lambda(J)=0$. Тогдас учетом леммы $1(5)$ получаем $M_{T} J=0$, где $M_{T}$ - идеал, порожденный в $T$ элементами $M$. В [4, теорема 1.4] доказано, что $T$ первично тогда и только тогда, когда $R$ $\varphi$-первично. Следовательно, $M_{T}=0$ (раз мы предположили $J \neq 0$ ) и $M=0$, т.е. $\lambda(J)$ - существенный правый идеал в $R$. По теореме Голди (см., например, [6, предложение 5.9]) в полупервичном правом кольце Голди каждый существенный правый идеал (в нашем случае $\lambda(J))$ содержит необратимый неделитель нуля, что противоречит лемме $1(6)$.

Заметим, что поскольку полупримитивное правое кольцо Голди не обязательно является первичным, условие $\varphi$-первичности кольца коэффициентов в теореме 1 не является необходимым. Так же $R$ не обязательно должно быть правьм кольцом Голди для того, чтобы $T$ было полупримитивным, например, если $R$ - кольцо формальных степенных рядов над полем от бесконечного числа переменных, то $J(T)=0$ по лемме $1(6)$.

ТЕОРема 2. Если кольцо $R$ ө-примитивно, то кольцо $T=R((x, \varphi))$ примитивно.

ДокАЗАТЕЛЬство. Пусть $M$ - максимальный правый идеал $R$, не содержаший нетривиальных $\varphi$-идеалов. Пусть $I$ - произвольный ненулевой идеал $T$. Тогда $\lambda(I)$ - ненулевой $\varphi$-идеал в $R$ по лемме $1(4)$, и, следовательно $\lambda(I)+M=R$. Теперь $I+\bar{M}=T$ по лемме $1(1)$, так как $1 \in \lambda(I+\bar{M})$. Таким образом, $\bar{M}$ не содержит ненулевых двусторонних идеалов, а из доказательства леммы 2 следует, что $\bar{M}-$ максимальный правый идеал $T$.

Полусовершенные кольца рядов Лорана. Для доказательства теоремы 3 нам будет полезна следующая характеризация полусовершенных колец.

Лемма 3 [8]. Кольчо $R$ полусовершенно тогда и только тогда, когда $R$ не содерхит бесконечного мнохсества ортогональных идемпотентов и любой элемент кольча $R$ есть сумма идемпотента и обратимого элеменma. 
ДОКАЗАТЕЛЬСТВо ТЕОРЕМЫ 3. Покажем, что в $T$ нет бесконечного множества ортогональных идемпотентов. Доказьвая “от противного", предположим, что $\left\{e_{i}=\sum_{k=t(i)}^{\infty} e_{k}^{(i)} x^{k} \in T \mid i=1,2, \ldots\right\}$ - такое множество. Обозначим $R / J(R)$ через $K$. В [5] было доказано, что над полупростым артиновым кольцом кольцо рядов Лорана будет полупростым артиновым, следовательно, $T / \overline{J(T)} \cong K((x, \varphi))$ полупростое артиново. Таким образом, $\left\{e_{i} \mid i=1,2, \ldots\right\}$ содержится в $\overline{J(T)}$ (кроме, возможно, конечного числа, но это не имеет значения). Для любого $i=1,2, \ldots$ из $e_{i}^{2}=e_{i}$ следует

$$
\left(e_{0}^{(i)}\right)^{2}+\underbrace{e_{-1}^{(i)} e_{1}^{(i)}+\cdots}_{\in J(R)}=e_{0}^{(i)},
$$

а из $e_{i} e_{j}=0$ при $i \neq j$

$$
e_{0}^{(i)} e_{0}^{(j)}+\underbrace{e_{-1}^{(i)} e_{1}^{(j)}+\cdots}_{\in J(R)}=0 .
$$

Кольцо $R$ полусовершенно, поэтому по [9, с. 120] счетное множество ортогональных идемпотентов (кольца $K)\left\{e_{0}^{(i)} \mid i=1,2, \ldots\right\}$ может быть поднято по модулю $J(R)$ до множества ортогональных идемпотентов кольца $R$. Но в полусовершенном кольце $R$ не может быть бесконечного множества ортогональных идемпотентов, следовательн, доказано, что в $T$ нет бесконечного множества ортогональных идемпотентов. Теперь с учетом следствия леммы 1 осталось воспользоваться характеризацией леммы 3 .

СлЕДСТВИЕ. Если кольцо $R$ полупримарно, то кольцо $T$ тожсе полупримарно.

ДоКАЗАТЕЛЬСТво. Полупримарное кольцо всегда является полусовершенным (идемпотенты в кольце поднимаются по модулю любого ниль-идеала $[9$, с. 118]), а по следствию леммы $2 J(T)$ нильпотентен.

Автор благодарит А. В. Михалёва за полезные советы и постоянное внимание к работе.

Московский государственный университет

Поступило им. М.В. Ломоносова 08.02 .96

\section{СПИСОК ЦИТИРОВАННОЙ ЛИТЕРАТУРЫ}

1. Мальцев А. И. // Докл. АН СССР. 1948. Т. 60. С. 1499-1501. 2. Risman L. // J. Pure Appl. Algebra. 1978. V. 12. P. 181-199. 3. Goodearl K., Small L. // Proc. Amer. Math. Soc. 1984. V. 92. P. 175-177. 4. Musson I., Stafford K. // Comm. Algebra. 1993. V. 21. № 6. Р. 2065-2075. 5. Сонин К.И. // Фундам. и прикл. матем. 1995. Т. 1. № 2. C. 565-568. 6. Goodearl K., Warfield R. An Introduction to Noncommutative Noetherian Rings. Cambridge: Cambridge Univ. Press, 1989. 7. Jordan C.R., Jordan D.A. // Comm. Algebra. 1976. V. 7. № 4. P. 647-656. 8. Camillo V., Hua-Ping Yu. // Comm. Algebra. 1994. V. 12. № 22. Р. 4737-4749. 9. Ламбек И. Кольца и модули. М.: Мир, 1971. 